\title{
The perforator-centralizing technique for super- thin anterolateral thigh perforator flaps: Minimizing the partial necrosis rate
}

\author{
Young Chul Suh, Na Rim Kim, Dai Won Jun, Jung Ho Lee, Young Jin Kim \\ Department of Plastic and Reconstructive Surgery, Bucheon St. Mary Hospital, College of Medicine, The Catholic University of Korea, \\ Bucheon, Korea
}

Background Despite the wide demand for thin flaps for various types of extremity reconstruction, the thin elevation technique for anterolateral thigh (ALT) flaps is not very popular because of its technical difficulty and safety concerns. This study proposes a novel perforatorcentralizing technique for super-thin ALT flaps and analyzes its effects in comparison with a skewed-perforator group.

Methods From June 2018 to January 2020, 41 patients who required coverage of various types of defects with a single perforator-based super-thin ALT free flap were enrolled. The incidence of partial necrosis and proportion of the necrotic area were analyzed on postoperative day 20 according to the location of superficial penetrating perforators along the flap. The centralized-perforator group was defined as having a perforator anchored to the middle third of the $x$ - and $y$-axes of the flap, while the skewed-perforator group was defined as having a perforator anchored outside of the middle third of the $x$ - and $y$-axes of the flap.

Results No statistically significant difference in flap thickness and dimension was found between the two groups. The arterial and venous anastomosis patterns of patients in both groups were not significantly different. Only the mean partial necrotic area showed a statistically significant difference between the two groups (centralized-perforator group, 3.4\% \pm $2.2 \%$; skewed-perforator group, $15.8 \% \pm 8.6 \% ; P=0.022$ ).

Conclusions The present study demonstrated that super-thin ALT perforator flaps can be elevated safely, with minimal partial necrosis, using the perforator-centralizing technique.

Keywords Free flaps / Perforator flaps / Microsurgical free flaps
Correspondence: Young Chul Suh Department of Plastic and Reconstructive Surgery, Bucheon St. Mary Hospital, College of Medicine, The Catholic University of Korea, 327 Sosa-ro, Wonmi-gu, Bucheon 14647, Korea

Tel: +82-32-340-2095

Fax: +82-32-340-7227

E-mail: remedios84@hotmail.com

Part of this article was presented at the 10th Congress of the World Society for Reconstructive Microsurgery on June 12-15, 2019, in Bologna, Italy.

\section{INTRODUCTION}

Wounds of the extremities are often shallow, and therefore require a stable soft tissue lining, with no need for dead space obliteration or a bulky flap. Even if the ultimate cosmetic out- come may not be the primary concern when reconstruction is performed, a functional flap contour remains necessary in order for the patient to wear well-fitting shoes and engage in unopposed motion of the wrist and ankle, as well as to prevent chronic flap friction or breakdown [1]. 
Despite the wide demand for thin flaps for various types of extremity reconstruction, the thin elevation technique for anterolateral thigh (ALT) flaps elevated above the superficial fascial plane is not very popular because of its technical difficulty and safety concerns.

Schaverien et al. [2] reported some anatomical limitations of the thinning technique. Their well-designed cadaveric study revealed a correlation between the extent of defatting and decreased flap vascularity. They described several types of perforator branching patterns of the lateral circumflex femoral artery and a venous branching pattern of the angiosome. In particular, the type 3 perforator complex (where the perforator divides into several branches at the suprafascial level, coursing horizontally for a varying distance before descending vertically to the subdermal plexus) significantly contributed to decreased vascularity when the flap was thinned.

Nakajima et al. [3] reported that the perforators of the lateral circumflex femoral vessel started at the deep adipofascial layer (DAL) and had a long running course at that level. Therefore, it is possible that not only the vascularity may be decreased by the thinning method, but also that the perforator of super-thin ALT flaps may have a skewed position after flap elevation. Kim et al. [4] reported that an eccentric position of the perforator did not have an impact on partial necrosis of the flap, but the impact of eccentricity in super-thin flaps has not yet been identified.

We performed a perforator-centralizing technique for superthin ALT flaps and analyzed its effects in comparison with a skewed-perforator group to propose a safe technique for elevating super-thin ALT flaps without partial necrosis.

\section{METHODS}

From June 2018 to January 2020, 41 patients who required coverage of various types of defects with a single perforator-based super-thin ALT free flap were enrolled. The study was approved by the institutional review board and the need for informed consent was waived due to the retrospective nature of the study.

Patient data were obtained retrospectively from the Bucheon St. Mary Hospital. We collected data regarding the demographic and clinical characteristics of the patients and the flaps. The following information about the reconstruction was also obtained: the pattern of microanastomosis, the occurrence of vessel insufficiency (artery or vein) or hematoma, the need for a revisional operation, the proportion of flap loss, and the duration of admission. The incidence of partial necrosis and the proportion of the necrotic area were analyzed on postoperative day 20 according to the location of superficial penetrating perforators along the flap. Group A (the centralized-perforator group) was defined as having a perforator anchored to the middle third of the $\mathrm{x}$ - and $\mathrm{y}$-axes of the flap, while group $\mathrm{B}$ (the skewed-perforator group) was defined as having a perforator anchored outside of the middle third of the $\mathrm{x}$ - and $\mathrm{y}$-axes of the flap (Fig. 1).

\section{Operative procedure for the perforator-centralizing technique}

All flaps were performed by a single surgeon (YCS). A technique for centralizing the superficial fascia penetrating perforator along the flap was initiated for the centralized-perforator group. First, the flap was designed with marking of all perforators to enhance

\section{Fig. 1. Illustration of group definitions}

In the centralized-perforator group (group A), the perforator was anchored to the middle third of the $x$ - and y-axes of the flap; in the skewedperforator group (group B), the perforator was anchored outside of the middle third of the $x$ - and $y$-axes of the flap.

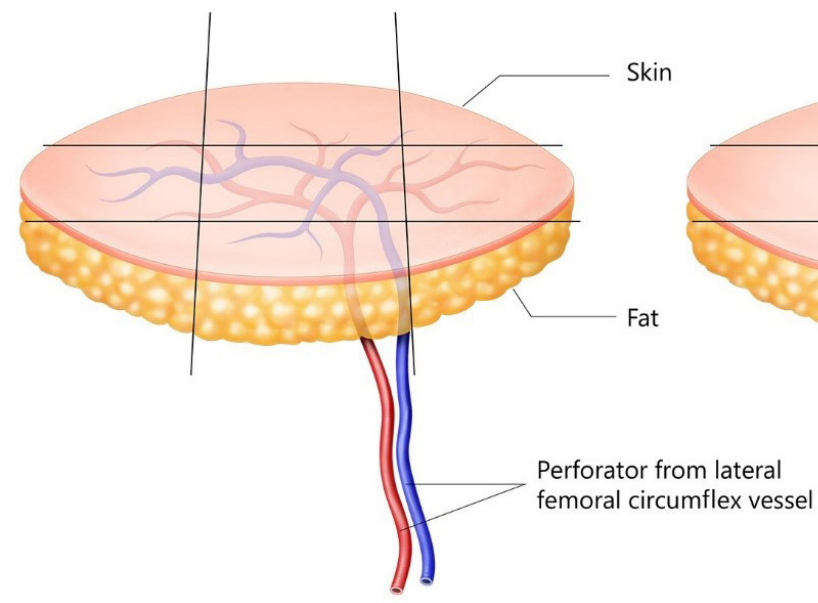

Group A
Group B 
the detection rate. The incision was made along only a quarter to half of the length of the entire designed line, and it extended down to the level of the superficial fascia. The superficial fascia is easily recognizable by the expansion of flap margin with a single self-retracting hook. Even if the superficial fascia does not seem to be definitively identifiable, the shape of fat lobules is extremely different between the superficial adipofascial layer and the DAL. The superficial fascia can be distinguished by the difference between large round fat cells in the deep layer and dense elliptical fat cells in the superficial layer. Once the perforator was identified at the superficial fascial level, the design could be rotated or shifted in order to centralize the perforator along the flap (Fig. 2). If the perforator was not in a skewed location, the flap was elevated according to the original design.

After harvesting the flap, the selected perforators were carefully dissected toward the source vessel through the remaining deep fat. The vascular pedicle was skeletonized and ligated at the takeoff of the rectus femoris branch; subsequently, the flap was prepared for microsurgical transfer to the recipient site.

\section{Statistical analysis}

Statistical significance for differences in demographic characteristics and complication rates was analyzed using SPSS version 19.0 (IBM Corp., Armonk, NY, USA). The Fisher exact test was used to compare categorical variables. The percentage of partial necrosis was calculated using ImageJ software (National Institutes of Health, Bethesda, MD, USA) using photos taken at 20 days postoperatively. The Mann-Whitney U test was done to determine the significance of differences in flap outcomes and operative factors between the centralized-perforator group and the skewed-perforator group. P-values $<0.05$ were considered to indicate statistical significance.

\section{RESULTS}

Of the 41 patients included, 26 patients had centralized perforators in the flap and 15 patients had skewed perforators. The mean age was 57.4 years (standard deviation [SD]: 6.7 years) in the centralized-perforator group and 59.1 years (SD: 7.4 years) in the skewed-perforator group. The mean body mass index (BMI) was $23.5 \mathrm{~kg} / \mathrm{m}^{2}\left(\mathrm{SD}: 2.39 \mathrm{~kg} / \mathrm{m}^{2}\right)$ in the centralizedperforator group and $22.8 \mathrm{~kg} / \mathrm{m}^{2}\left(\mathrm{SD}: 3.03 \mathrm{~kg} / \mathrm{m}^{2}\right)$ in the skewed-perforator group. The mean age and BMI did not show significant differences between the two groups. There were no statistically significant between-group differences in the prevalence of hypertension $(P=0.52)$, diabetes mellitus $(P=0.103)$, previous smoking $(\mathrm{P}=0.272)$, current smoking $(\mathrm{P}=1.000)$, peripheral vascular disease $(\mathrm{P}=0.318)$, and coronary heart disease ( $\geq 2$-vessel disease) $(\mathrm{P}=0.101)$ (Table 1$)$.

There was no statistically significant difference in flap thickness or dimensions between the two groups. The arterial and venous anastomosis patterns of the patients in both groups were not statistically different. The incidence of flap failure was zero in both groups and the incidence of revisional microsurgery, wound dehiscence, and skin graft to the partial necrotic area likewise

Table 1. Patients demographic, and comorbidities

\begin{tabular}{|c|c|c|c|}
\hline Variable & $\begin{array}{c}\text { Perforator } \\
\text { centralized } \\
\text { group } \\
(n=26)\end{array}$ & $\begin{array}{l}\text { Perforator } \\
\text { skewed } \\
\text { group } \\
(n=15)\end{array}$ & P-value \\
\hline \multicolumn{4}{|l|}{ Defect location } \\
\hline Lower extremity & 25 & 13 & \\
\hline Upper extremity & 1 & 0 & \\
\hline Head and neck & 0 & 2 & \\
\hline Age (yr) & $57.4(2-72)$ & $59.1(24-81)$ & 0.681 \\
\hline $\mathrm{BMI}\left(\mathrm{kg} / \mathrm{m}^{2}\right)$ & $23.5(21.3-29.6)$ & $22.8(20.4-31.3)$ & 0.821 \\
\hline \multicolumn{4}{|l|}{ Comorbidity } \\
\hline Hypertension & $12(46.1)$ & $9(60.0)$ & 0.520 \\
\hline Diabetes mellitus & $15(57.7)$ & $4(26.7)$ & 0.103 \\
\hline Previous smoker & 5 (19.2) & $6(40.0)$ & 0.272 \\
\hline Current smoker & $3(11.5)$ & $2(13.3)$ & $>0.999$ \\
\hline Peripheral vascular disease & 12 (33.3) & $4(26.7)$ & 0.318 \\
\hline $\begin{array}{l}\text { Coronary heart disease } \\
(\geq 2 \text { VD) }\end{array}$ & $12(33.3)$ & $3(20.0)$ & 0.101 \\
\hline
\end{tabular}

Values are presented as mean (range) or number (\%).

BMI, body mass index, VD, vessel disease.

\section{Fig. 2. Perforator-centralizing technique}

(A) After identifying the perforator at the superficial fascial level, (B) the design can be rotated or shifted in order to centralize the perforator along the flap.
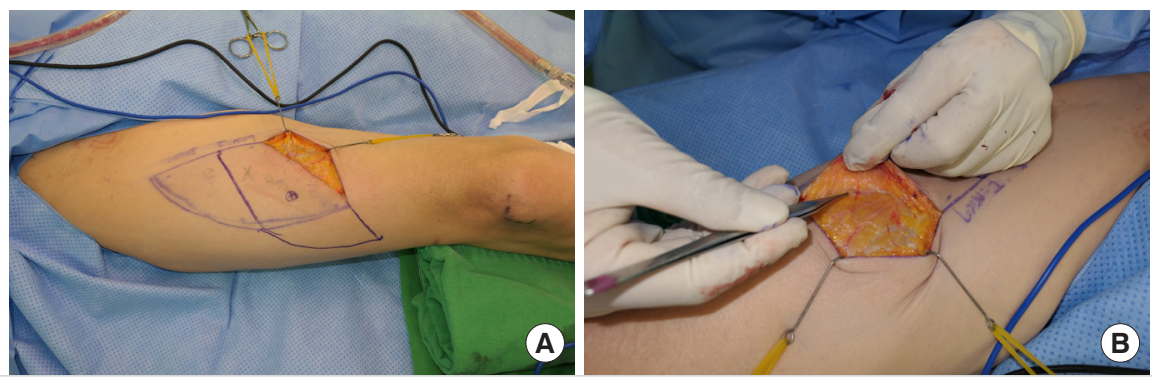
Table 2. Comparison of flap outcome and partial necrosis rate between perforator centralized group and perforator skewed group

\begin{tabular}{|c|c|c|c|}
\hline Variable & $\begin{array}{l}\text { Perforator centralized group } \\
\qquad(\mathrm{n}=26)\end{array}$ & $\begin{array}{l}\text { Perforator skewed group } \\
\qquad(n=15)\end{array}$ & P-value \\
\hline Flap thickness (mm) & $6.7(4.2-9.5)$ & $7.0(4.5-10.2)$ & 0.591 \\
\hline Flap length $(\mathrm{cm})$ & $12.5(3.2-21.0)$ & $14.3(3.0-19.0)$ & 0.359 \\
\hline Flap width $(\mathrm{cm})$ & $6.7(1.5-11.5)$ & $6.3(2.0-9.5)$ & 0.813 \\
\hline \multicolumn{4}{|l|}{ Arterial microanastomosis pattern } \\
\hline End-to-end & $10(38.5)$ & $8(53.3)$ & 0.515 \\
\hline End-to-side & $16(61.5)$ & $7(46.7)$ & 0.515 \\
\hline \multicolumn{4}{|l|}{ Venous microanastomosis pattern } \\
\hline 1 Vein & $25(96.1)$ & $15(100)$ & 0.442 \\
\hline 2 Veins & $1(3.9)$ & 0 & \\
\hline Flap failure & 0 & 0 & \\
\hline Revisional microsurgery & $2(7.7)$ & 0 & 0.524 \\
\hline Wound dehiscence & $3(11.5)$ & $2(13.3)$ & 0.271 \\
\hline Flap elevation time (min) & $72.5(45.5-81.0)$ & $64.7(47.0-78.0)$ & 0.312 \\
\hline Partial necrosis & $6(23.1)$ & $3(19.8)$ & \\
\hline Skin graft to partial necrosis area & $2(7.7)$ & $1(6.5)$ & 0.903 \\
\hline Secondary healing for partial necrosis area & $4(15.4)$ & $2(13.3)$ & 0.858 \\
\hline Partial necrosis area (proportion to whole flap area) (\%) & $3.4(3.1-10.7)$ & $15.8(11.5-48.5)$ & $0.022^{\mathrm{b})}$ \\
\hline Flap length of the subgroup $(\mathrm{cm})$ & $13.7(11.5-17.0)$ & $15.2(9.5-20.0)$ & 0.762 \\
\hline Flap width of the subgroup $(\mathrm{cm})$ & $7.8(5.0-8.5)$ & $6.0(4.0-7.5)$ & 0.425 \\
\hline
\end{tabular}

Values are presented as mean (range) or number (\%).

a)Average (range); ${ }^{\text {b) }}$ Statistical significance was set at $P<0.05$.

\section{Fig. 3. Case 1}

An 80-year-old man showed malignant melanoma on the sole of his left foot (A). A super-thin anterolateral thigh flap measuring $16 \times 5 \mathrm{~cm}^{2}$ was used to cover the defect. On postoperative day 20, a necrotic area of $17 \%$ was noted at the distal part of the flap (B-E). The eschar was debrided and the defect was covered with a full-thickness skin graft.
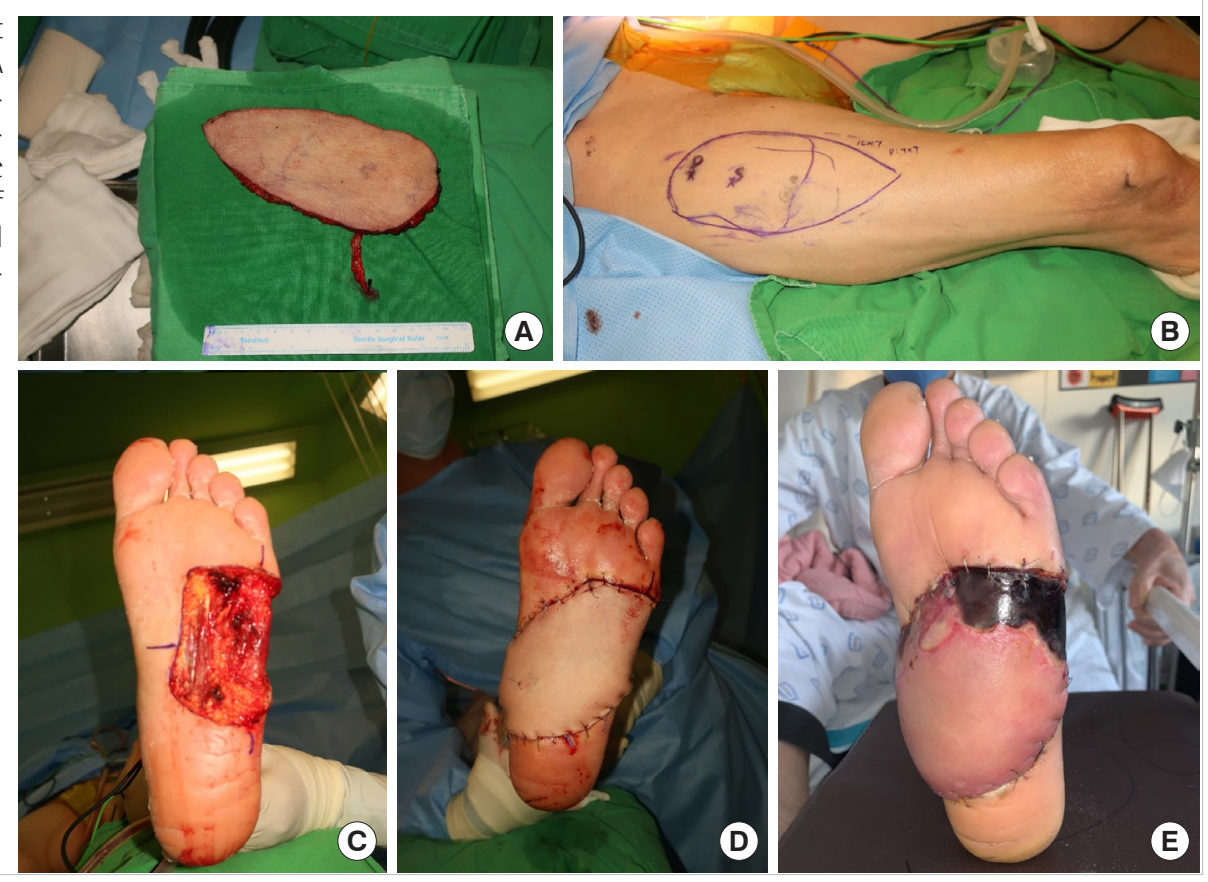

showed no statistically significant differences between the two groups. The mean size of the partially necrotized flaps in both groups did not show a statistically significant difference. Only the mean partial necrotic area was significantly different between the groups (centralized-perforator group, $3.4 \% \pm 2.2 \%$; skewedperforator group, $15.8 \% \pm 8.6 \% ; \mathrm{P}=0.022)$ (Table 2$)$.

\section{Case reports}

Case 1

An 80-year-old man had malignant melanoma on the sole of his left foot (Fig. 3A). A super-thin ALT flap measuring $16 \times 5 \mathrm{~cm}^{2}$ was used to cover the defect. On postoperative day 20, a necrotic area of $17 \%$ was noted on the distal part of the flap (Fig. 3B-E). 


\section{Fig. 4. Case 2}

A 47-year-old man had a traumatic soft tissue defect with plate exposure on his right medial malleolus area (A). A $15 \times 9 \mathrm{~cm}^{2}$ super-thin anterolateral thigh flap was used to cover the defect. The perforator was encountered more distally, so the design was rotated in order to centralize the perforator (B-E). The flap successfully healed without any necrosis at postoperative 6 months (F).
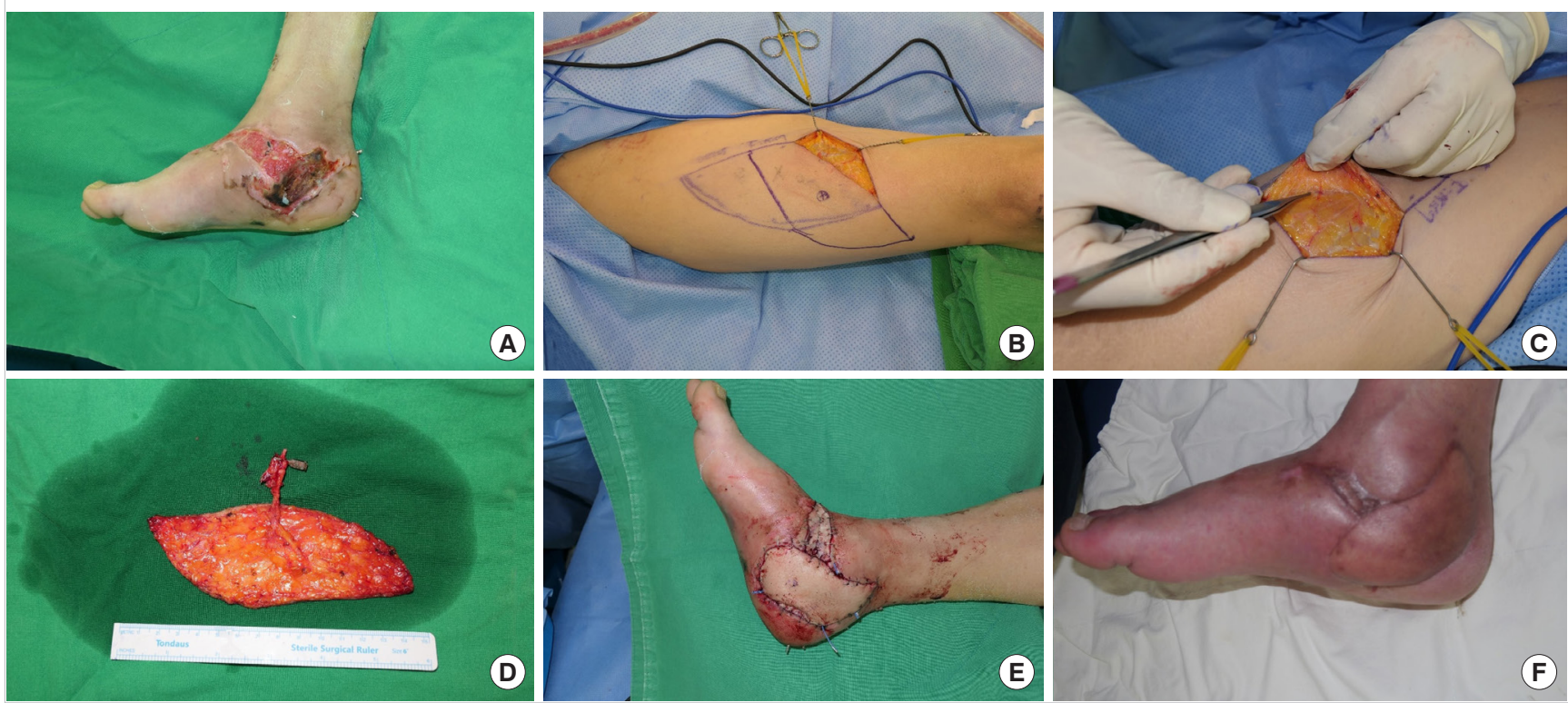

The eschar was debrided and the defect was covered with a fullthickness skin graft.

\section{Case 2}

A 47-year-old man had a traumatic soft tissue defect with plate exposure on his right medial malleolus area (Fig. 4A). A $15 \times 9$ $\mathrm{cm}^{2}$ super-thin ALT flap was used to cover the defect. The perforator was encountered more distally, so the design was rotated in order to centralize the perforator (Fig. 4B-E). The flap successfully healed without any necrosis at postoperative 6 months (Fig. 4F).

\section{DISCUSSION}

Traditionally, during preoperative planning, the microvascular anatomy of the perforator is only studied to determine where it emerges from the muscular fascia, as well as its caliber and velocity, using both color Doppler ultrasonography and computed tomography angiography.

However, Nakajima et al. [3] analyzed the perforator patterns of the lateral circumflex femoral artery, and revealed that these perforators arborize and run transversely for a long distance inside the DAL. The vessel starts in the DAL and connects to either the direct cutaneous branch or the perforating cutaneous branch of muscular arteries. Therefore, if we can successfully capture the perforator penetrating the deep fascia, it is important for thinning the ALT flap both to preserve this horizontally running course in the DAL and to centralize the superficial anchoring perforator.

Only a few groups have reported techniques for super-thin ALT flaps $[1,5,6]$. In previous explanations, the super-thin ALT elevation technique was limited to identification of the superficial fascia, not the vascular anatomy along the plane. Technical difficulties arose from unexpectedly encountering perforators along the superficial fascial level. The uncertainty of the actual location of the superficial fascial-penetrating perforator contributes to the elevation of the eccentric type of perforator flap, as in the skewed-perforator group. This issue results in underestimating the super-thin ALT flap compared to other kinds of thinning techniques, such as primary defatting and microdissection [7].

Some studies have investigated the safety and reliability of eccentrically positioned perforator flaps $[4,8]$. The perforasome/ angiosome theories point out that the flap axis should be based on the direction of the linking vessels [2]. In the perforasome of the main source artery, large linking vessels course parallel to the muscle fibers. For this reason, the axis of the perforator follows the orientation of the muscle fibers. Therefore, if the flap design and skin paddle orientation follow this axis, the vascularity of the flap would align with the axis of the perforator. Under such circumstances, even if the perforator is located at the margin of the flap, it would still flow along this axis and perfuse the entire flap [4].

However in super-thin ALT flaps, the axis of flap is less meaningful because the linking vessel is not contained in the flap vas- 
culature. In this study, the position of the perforator was found to be an important factor affecting the size of the partial loss area (centralized-perforator group, $3.4 \% \pm 2.2 \%$; skewed-perforator group, $15.8 \% \pm 8.6 \% ; \mathrm{P}=0.022$ ) (Table 2). On average, a length of 12-14 cm and a width of 6-7 cm can be safely elevated for a super-thin ALT flap using the perforator-centralizing technique. Furthermore, based on the subgroup analysis of partially necrotized flaps, if the flap length and width exceed $9 \mathrm{~cm}$ and $4 \mathrm{~cm}$, respectively, we recommend using this technique for super-thin ALT flap elevation.

Various kind of thinning methods have been suggested, although studies have only assessed changes in the perforasome based on flap thickness $[2,6,7,9,10]$. This study was conducted to minimize partial necrosis along the super-thin flaps not based on their thickness, but based on the location of the perforator that can accidentally be captured at the distal margin of the flap. Of course, additional effort is needed to elevate a super-thin ALT flap rather than the classical perforator flap, and this includes dissection of the perforator above the deep fascial plane and capturing the superficial fascia-anchored point in the middle of the flap. Nonetheless, this surgical technique improves the safety of super-thin ALT perforator flaps.

A limitation of this study is that we could not assess partial flap loss due to venous congestion. In general, partial flap loss can be caused by either angiosomal or venosomal problems, but in this study, partial necrosis was classified as a single problem, so the impact of venous complications may have been underestimated.

\section{NOTES}

\section{Conflict of interest}

No potential conflict of interest relevant to this article was reported.

\section{Ethical approval}

The study was approved by the Institutional Review Board of Bucheon St. Mary Hospital (IRB No. HC2021-0085-0001) and performed in accordance with the principles of the Declaration of Helsinki.

\section{Patient consent}

The patients provided written informed consent for the publication and the use of their images.

\section{Author contribution}

Conceptualization: YC Suh, NR Kim, DW Jun, JH Lee, YJ Kim. Data curation: YC Suh. Formal analysis: YC Suh. Methodology:
DW Jun, JH Lee. Project administration: YC Suh. Visualization: YC Suh. Writing - original draft: YC Suh. Writing - review \& editing: YC Suh, NR Kim.

\section{ORCID}

Young Chul Suh https://orcid.org/0000-0002-0320-3933

Na Rim Kim https://orcid.org/0000-0002-6046-4566

Dai Won Jun https://orcid.org/0000-0003-4713-333X

Jung Ho Lee https://orcid.org/0000-0002-3800-5494

Young Jin Kim https://orcid.org/0000-0002-9046-9907

\section{REFERENCES}

1. Seth AK, Iorio ML. Super-thin and suprafascial anterolateral thigh perforator flaps for extremity reconstruction. J Reconstr Microsurg 2017;33:466-73.

2. Schaverien M, Saint-Cyr M, Arbique G, et al. Three- and four-dimensional computed tomographic angiography and venography of the anterolateral thigh perforator flap. Plast Reconstr Surg 2008;121:1685-96.

3. Nakajima H, Minabe T, Imanishi N. Three-dimensional analysis and classification of arteries in the skin and subcutaneous adipofascial tissue by computer graphics imaging. Plast Reconstr Surg 1998;102:748-60.

4. Kim YH, Lee HE, Lee JH, et al. Reliability of eccentric position of the pedicle instead of central position in a thoracodorsal artery perforator flap. Microsurgery 2017;37:44-8.

5. Hong JP, Choi DH, Suh H, et al. A new plane of elevation: the superficial fascial plane for perforator flap elevation. J Reconstr Microsurg 2014;30:491-6.

6. Kim SY, Lee YJ, Mun GH. Anatomical understanding of target subcutaneous tissue layer for thinning procedures in thoracodorsal artery perforator, superficial circumflex iliac artery perforator, and anterolateral thigh perforator flaps. Plast Reconstr Surg 2018;142:521-34.

7. Agostini T, Lazzeri D, Spinelli G. Anterolateral thigh flap thinning: techniques and complications. Ann Plast Surg 2014;72:246-52.

8. Ulas Bali Z, Kececi Y, Pabuscu Y, et al. Anterolateral thigh flap design by using suprafascial course of the perforator. Ann Plast Surg 2020;84:550-3.

9. Kimura N, Satoh K, Hasumi T, et al. Clinical application of the free thin anterolateral thigh flap in 31 consecutive patients. Plast Reconstr Surg 2001;108:1197-208.

10. Kimura N, Satoh K, Hosaka Y. Microdissected thin perforator flaps: 46 cases. Plast Reconstr Surg 2003;112:1875-85. 